\section{PET and PET/CT Study Guide: A Review for Passing the PET Specialty Exam}

\author{
A. Moniuszko and A. Sciuk
}

New York, NY: Springer, 2013, 240 pages, $\$ 39.95$

PET and PET/CT Study Guide will appeal to an audience of physicians, residents, educators, technologists, and students wishing to acquire basic and advanced knowledge of PET and PET/CT in preparation for certifying boards or for working in PET and PET/CT laboratories. The study guide was designed not to assess knowledge but to serve as a practical tool for developing and enhancing the skills needed to perform PET and PET/CT in the clinical setting.

The study guide contains 5 chapters and a section of appendices. Four of the chapters contain test questions. The appendices contain supporting information and can assist the reader in preparing to sit for a certifying examination or in building a knowledge base on PET and PET/CT.

Chapter 1 begins with a useful discussion on test strategies. Successfully navigating a multiple-choice test requires a basic understanding of the question's format, including the stem, options, and distractions. The author provides a variety of ways to examine each question in terms of correctly identifying the main components of what is being asked, discarding the portions of the question that are irrelevant, recognizing which options can be quickly eliminated, and determining how to make the final selection-even if that final selection is a guess. Additionally, a brief discussion of test anxiety is provided, with emphasis on creating a positive study plan and environment, preparing physically and mentally for the test day, and using positive affirmations for a successful outcome.

Chapters 2 through 4 contain practice tests with ascending degrees of difficulty, from "easy" to "hard." Each chapter contains a 200-question examination, correct answers with references, and, in most cases, a brief explanation of the topic or response. Chapter 5 contains an abbreviated test with an additional 50 bonus questions, answers, and again, in most cases, a brief explanation of the response. These test chapters provide the reader with an excellent experience in preparing for and taking an examination, identifying areas that may require additional study, and practicing the many manual calculations and computations that may have been lost over years of using today's modern technology.
The author describes the testing pathway from easy to hard as a building block that can assist the reader in constructing a solid base of knowledge and in completing subsequent examinations or working in the clinical setting.

Of the 4 appendices, appendix A is perhaps the most important in preparing for a national certification examination. This appendix contains some necessary formulas, numbers, and conversion rates that can be quickly forgotten in today's clinical environment, in which automated technology makes calculations and conversions with minimal interaction from the user. A review of this appendix is required reading for most to be successful in the calculations and computations that are sure to be found on the PET examination. Appendix B provides a list of commonly used abbreviations and symbols in nuclear medicine; appendix $\mathrm{C}$, a limited glossary of terms; and appendix D, a useful list of Web sites containing supporting information for PET and PET/CT.

Although the book is described as a study guide, the reader is required to use the references to obtain additional information on the topics covered, given that the format includes the test questions but only minimal information in the answer section. In general, reference materials are current and readily available and will provide the reader with a comprehensive discussion of the topics.

PET and PET/CT Study Guide is a useful tool for those preparing to sit for the NMTCB certifying examination on positron emission or for those working in a PET or PET/CT department. The study guide allows the reader to assess personal knowledge using a test format that mirrors the NMTCB PET content outline. The easy-to-hard test format and the references for additional reading will allow the reader to enhance personal knowledge of PET and PET/CT, develop a successful study plan to augment areas of deficiency, and be successful in completing the national certifying examination or in working in today's busy PET or PET/ CT laboratory.

\author{
Kathy Thomas, MHA, CNMT \\ Capintec, Inc. \\ 10 SE 13th St. \\ Battle Ground, WA 98604 \\ E-mail: ksthomas0412@msn.com
}

Published online Oct. 21, 2013.

DOI: $10.2967 /$ jnmt.113.132183 